Informatics and Technology | Igor Smolyar, Martin Wikelski, Tim Bromage

\section{Quantifying layered patterns in nature, medicine, and materials}

The morphology of layered anisotropic systems plays a crucial part in the development of new materials as well as in medical, forensic and biomime research. The ability to quantitatively describe these morphologies is from the National Centers for Environment Information. Dr Igor Smolyar, Tim Bromage from New York University and Professor Dr Marti Wikelski from the Max Planck Institute of Animal Behavior and Univer. of Konstanz, have developed an empirical model that can quantify the structural characteristics of layered patterns.

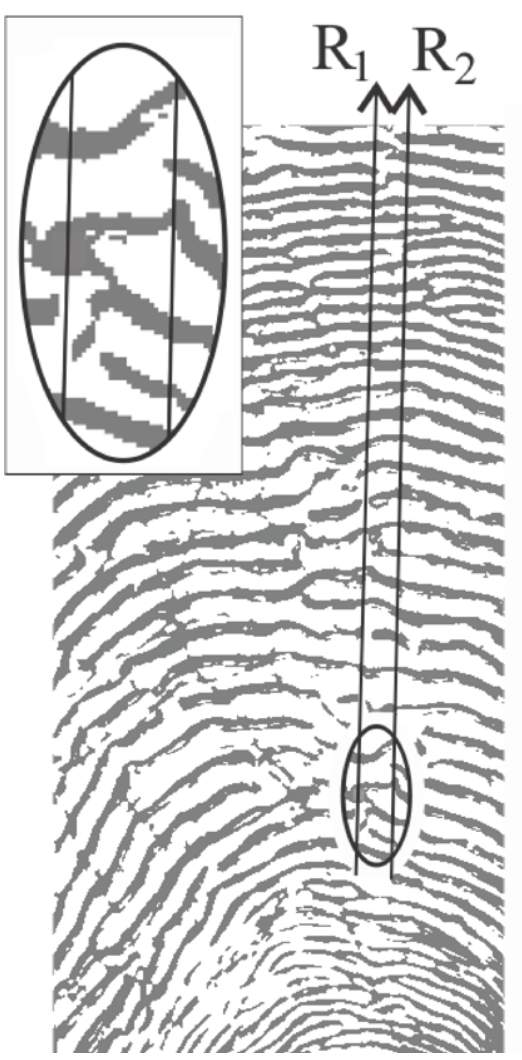

Waturally occurring structures, Wuch as sand dune ripples, and lamellae in vertebrate bones, are made up of systems of layers. Many manmade materials, including alloys, polymers and ceramics, also comprise layered structures. These land external factors that roguth the formation of the patterns. The form and structure of these layers make it possible to identify trends periodicities, and events in the history of the formation of these systems. This morphology of layered systems plays a crucial part in the developme of new materials as well as medical forensic and biomimetic research.

Aspects of layered system analysis still need to be formalised for solving scientific and engineering problems. The ability to quantitatively describe is required if this go lased pattern is required if this goal is to be achieved. however, due to the myrematic, confluences and bifurcations that occur in the layers of two-dimensional epresentations of three-dimensional objects. The number and thickness vary depending on which direction they are measured in; this makes the development of a formal analysis procedure difficult.

Dr Igor Smolyar, currently with the National Centers for Environment Information/NOAA, Professor Tim Bromage, director of the Hard Tissue Research Unit at New York University and Professor Dr Martin Wikelski, founding director of the Max Planck professor at Konstanz University, are addressing this problem. They have developed an empirical model (EM) that can be used for quantification of the structural characteristics of layered anisotropic patterns.

\section{FISH SCALES}

The research team initially explored the patterns of fish scales. They chose this starting point as the information gleaned from fish scales enabled them to address broader issues within marine science.

The centre of a fish scale, the focus, represents the tiny scale of the new ciched fish. The scales develop as the fish grows, like the rings in a of these layers are 'anisotropic' in ee trunk. These growth increments form layers that are referred to as hat are constructed from the circul areation regarding the eve in the fish's life history and the state of their habitat. The research team found their fish scale
research impeded as some steps that they required for their analysis had not yet been formalised. The results of the analytical processing of fish scales are subject to the investigator's perceptions and preconceptions. Furthermore, ther are inherent problems in formalising the procedures and parameterisation of fish scales due to incremental patter anisotropy, where the size and number of circuli are functions of the dire of measure funt. The hythmical lement of the carameterisation procedure and set about developing method for the quantification of rhythmical structures that would take anisotropy into accoun

\section{QUANTIFICATION OF}

ANISOTROPIC GROWTH

The anisotropic growth of fish

scales means that the structure of the growth increments has to be analysed in various directions in the 2D plane. The researchers translated the anisotropic structure of fish scale patterns into N-partite graphs, made up ofverices and edges. Thickness of the growth increment along each transect, rcaction, is proporithal to the fish (low the team to introduce the notion of "layer structure" across the 2D plane and fully formalised construction

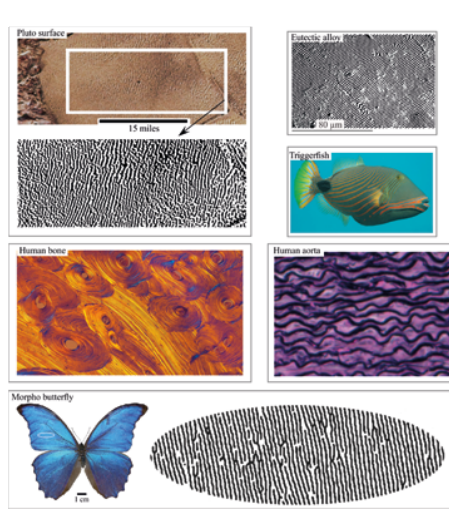

Figure 3. Layered anisori
nature and beyond.

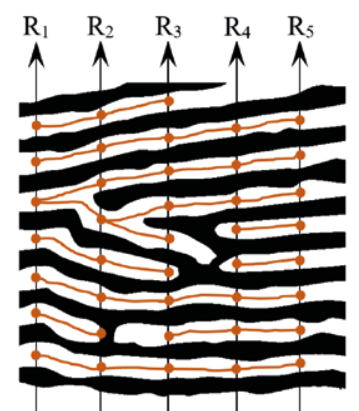

From fish scale pattern to

N-partite grap

Figure 2. Formalisation of a
layered anisotropic structure

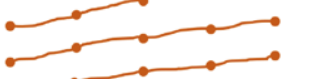

The form and structure of these layers make it possible to identify trends,

periodicities, and events in the history of pattern formation.

of "layer thickness vs layer number" and "layer area vs layer number"

the time it was formed, so a layer's thickness and area provide the growth rate variability of the layered system at that time. These charts enable the researchers to describe the morphological characteristics
of an entire 2D layered pattern.

Due to circuli anisotropy, various layer structures are possible across the 2D plane, resulting in uncertainty in the quantification of morphological features of layered anisotropic patterns. thas been shown that describing the with confidence and high-level accuracy are a mutually exclusive goal. Uncertainty quanting anisotopic layed paten

THE EMPIRICAL MODEL (EM) The N-partite graphs, Boolean along transects make up the EM that formalises the anisotropy of the structure and size of layered systems.

Numerous natural forms are made up of layers with structures similar to those of fish scales. Satellite images provide evidence of large-scale patterns on Earth and other planets. (n) in corals, marine and terrestrial sand Smallscall as sediment profiles. SThe amellar corpuscles in humans and nimals as well as the macro structure of buts including alloys, perlite steels,

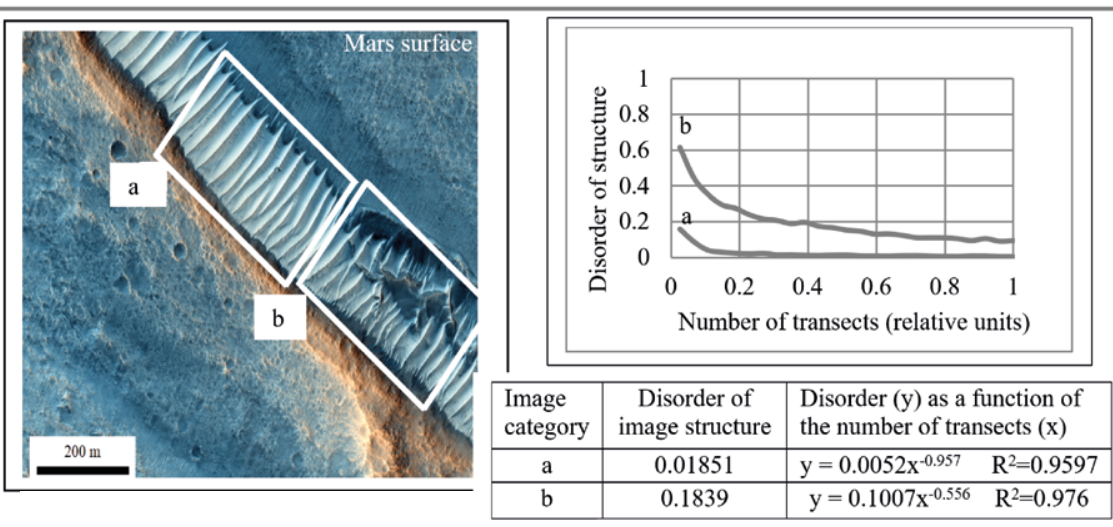

Figure 4. Structural anomaly of a Mars surface layered systen
Credit: NASA/JPL/Univ. of Arizona. PSP_008641_2105 

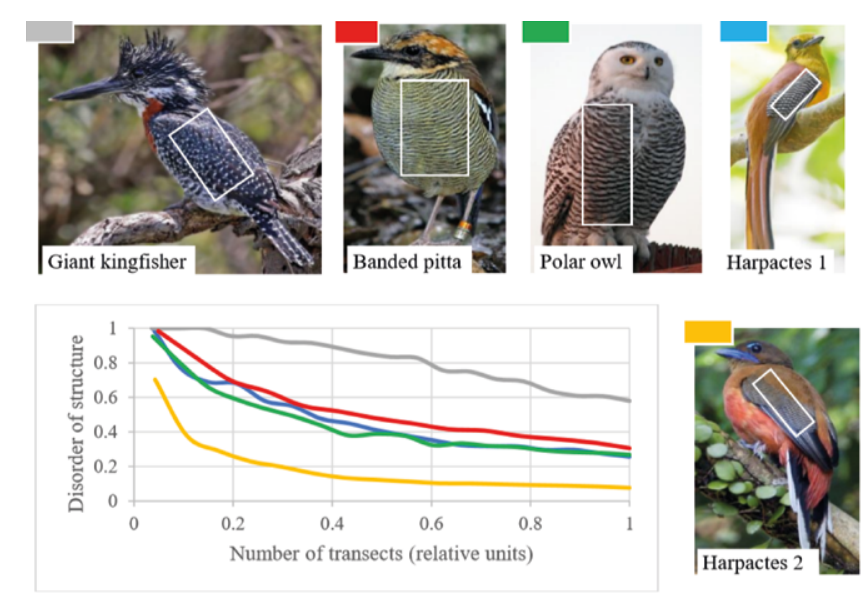

. Figure 6 (below). Examples of arbitrary patterns for future research.
Credits: Melanoma: Wikikedia contributor; Dragonfly wings: Iron Chris, Credits: Melanoma:
Wikipedia contributor
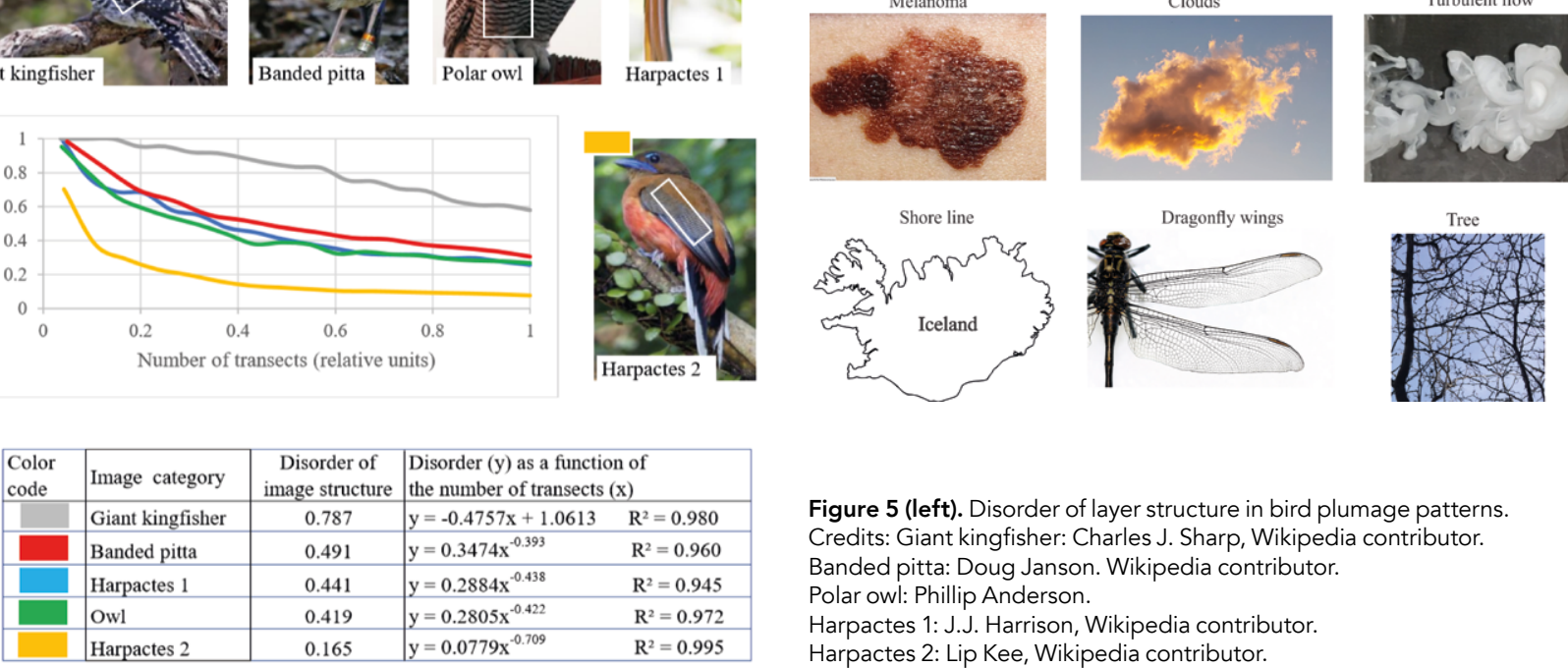

\section{Figure 5 (left). Disorder of layer structure in bird plumage patterns.} Credits: Giant kingfisher: Charles J. Sharp, Wikipedia contributor. Banded pitta: Doug Janson. Wikipedia contributor. Horractes 1: I.J.J Harrison, Wikipedia contributor.
Harpactes 2: Lip Kee, Wikipedia contributor.

and structure. The layered anisotropic and external factors that regulate the formation of patterns in both these ving and non-living systems.

\section{DISORDER OF LAYER STRUCTUR} The research team has shown that their EM can be applied to layered patterns egardless of their size and origin. They have recently introduced a new structura characteristic, disorder of layer structure (DStr) that measures the deviation of a $2 \mathrm{D}$ layered pattern from isotropy. Isotropy efers to uniformity in all directions in contrast with direction-dependa have have both isotropic and anisotropic from zero when ther is minimal disorder to ne where there is maximal disorder. DStr can also be used to differentiate between layered and non-layered patterns on both a local and global scale. The researchers have shown that DStr is a universal characteristic that can be applied to any $2 \mathrm{D}$ layered patterns demonstrating its use with geological atmospheric, medical, material, forensic, plant, and animal images.

The research team has demonstrated how DStr can be used to uncover structural anomalies in anisotropic layered patterns by monitoring and ripples on the sund dunes and Mars and examining the struct anomaly. They have also formulated testable hypotheses by studying the correspondence between physical, mechanical, and biological properties

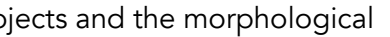
characteristics of their layered patterns.

\section{AREAS OF APPLICATION} Analysing the growth-rate variability of lamella bones with EM allows the researchers to reveal cyclicity in bone formation that had not previously discovered that lamellar increments of

bivalve shells, are functions of seawater parameters. EM could be used to support the study of local and globa climatic characteristics of the world ocean and its dynamics.

Birds' morphology could be analysed using EM and together with their migration patterns across the oceans, marine data from the World Ocean Database and the International Comprehensive Ocean-Atmosphere Data Sets, this could provide a

\section{The layered anisotropic patterns}

form a record of the internal and external factors that regulate the formation

\section{of patterns.}

bone and growth increments of tooth enamel are formed at the same time. These hard tissue rhythms provide events occurring during bone and tooth formation and are of significant interest to mammal life history researchers.

The research team has a particular interest in the link between growth increments and the marin environment. Morphological charactenstics of the growth increments ocean, such as fish scales, corland of testable hypotheses to inform protection, and research into the spread of infectious diseases.

M can be used to quantitatively describe the relationship between structure and properties of a layered system. It provides a formal process structural anomalies that could solve fundamental problems in the study chlayered objects and prove a positive science, and biomimetic research. to track structural changes and reveal

\section{Behind the Research a Igor Smolyar

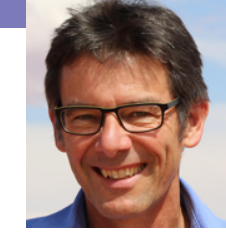 \\ Martin \\ Wikelski \\ W: https://www.ab.mpg.de/ W: https://www.ab.mpg.de/wikelsk

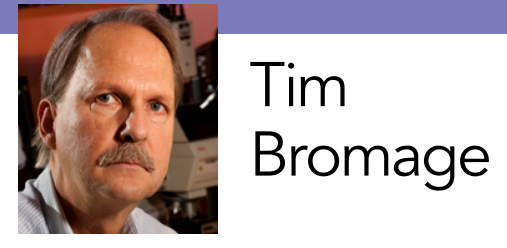 \\ http://dental.nyu.edu/faculty/ft/ tgb3.htm \\ Research Objectives}

The team of researchers used fish scales and lamellar bones to develop an empirical model for the quantification of layered anisotropic patterns.

\section{Detail}

Bio

Prof Martin Wikelski studied biology University of Washington in Seattle and the Smithsonian tropical research Institute in Panama. Martin continued s assistant professor at the University of lllinois in Urbana-Champaign, then became assistant and associate professor tPrinceton University. He then returned to Germany to become the founding

animal behaviour and professor at he University of Konstanz. He is a member of the German Academy of sciences Leopoldina.

Professor Tim Bromage directs the Hard Tissue Research Unit, a technology development laboratory of New York University. Recently, they reported on a chronobiological rhythm in hard
He is a recipient of the 2010 Max Planck Prize in the Life Sciences for this work. Igor Smolyar graduated from Polytechnical Institute in Russia and received a PhD in computer science Currently within the National Centers for Environmental Information/NOAA his area of interest is the application of discrete methods for the study of natur history phenomena.

\section{References}

Smolyar I., Bromage T., Wikelski M. (2019). Layered pattern in nature, medicine, and materials: quantifying anisotrop

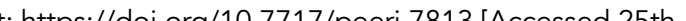
November 2019].

Smolyar I., Bromage T., Wikelski M. (2016). Quantification of layered patterns with structural anisotropy: a comparison of biological and geological systems. Heliyon, Lonline heliyon.2016.e00079 [Accessed 25th November 2019].

Smolyar I.V. (2014). System and Method for Quantification of Size and Anisotropic Structure of Layered Patterns. U.S. Patent 8,755,578, issued June 17, 2014 .

\begin{tabular}{ll|l}
$\mathbb{1}$ & NYU & COLLEGE \\
OF DENTISTRY
\end{tabular}

\section{Personal Response}

\section{What are your plans for future research into the} morphology of layered patterns?

We intend in the future to investigate the applicability of our empirical model for processing categories of patterns beyond the layered patterns discussed here. Examples of in Figure 6. To reach our goal we plan to empirically justify that there is similarity between layered and arbitrary patterns allowing us to use EM to formalise the structure
and size of arbitrary patterns. Objects of our study will be binary and grayscale arbitrary patterns, both formed in nature and manmade.

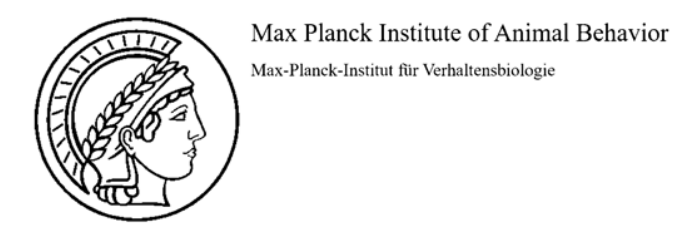

